\title{
Characterization of Path-Loss Disparity in Virtual Multiple-Input-Single-Output Links
}

\author{
Haejoon Jung and Mary Ann Weitnauer \\ Department of Electrical and Computer Engineering, Georgia Institute of Technology, Atlanta, GA 30332, USA \\ Correspondence should be addressed to Haejoon Jung; h.j.jung@ieee.org
}

Received 7 March 2014; Revised 21 June 2014; Accepted 22 June 2014; Published 21 July 2014

Academic Editor: Theodoros Tsiftsis

Copyright ( 2014 H. Jung and M. A. Weitnauer. This is an open access article distributed under the Creative Commons Attribution License, which permits unrestricted use, distribution, and reproduction in any medium, provided the original work is properly cited.

\begin{abstract}
Cooperative transmission (CT) is an effective technique to achieve spatial diversity in fading environments, where spatially separated wireless nodes collaborate to form a virtual antenna array or virtual multiple-input-multiple-output (VMISO) link. Many authors model the path loss of the VMISO link as though the elements in the virtual antenna array are colocated, even though they are spread out. In this paper, we show that the spreading causes a signal-to-noise-ratio (SNR) penalty of up to $3 \mathrm{~dB}$. Moreover, in the high SNR regime, we show that the performance degradation caused by the path-loss disparity can be characterized equivalently by log-normal distribution. We use these two observations to propose a new CT link model, expressed in closed form as an outage probability based on the log-normal shadowing model. The quality of the model is evaluated by a statistical test based on Kolmogorov-Smirnov method.
\end{abstract}

\section{Introduction}

For small wireless nodes with limited power, where collocated antennas (a real antenna array) cannot be deployed, CT is an alternative way to achieve spatial diversity in fading channels $[1,2]$. CT provides an SNR advantage through array and diversity gains by creating a VMISO link that connects a transmitting cluster (multiple nodes) with a single receiver node. Based on the SNR advantage of CT, various higher layer protocols have been proposed, in which the VMISO links provide gains at higher layers such as throughput improvement, energy saving, energy balancing, and range extension [3-16].

In multihop ad hoc networks, the CT-based protocols are divided into two (decentralized and centralized architectures) depending on how the cooperative clusters are activated and supported [17]. In the decentralized architecture such as [38], the cluster is activated randomly in a distributed manner, where all the nodes in a cluster have an equal role. On the other hand, in the centralized cluster architecture, which has a cluster head in each cluster, the cluster head recruits its cooperators and triggers the group transmission to the cluster head in the next cluster [12-16]. Because the member nodes follow their cluster heads' decision and the cluster size can be readily controlled, a cluster can work as a single node. Therefore, the centralized cluster architecture is highly compatible with the conventional non-CT or single-inputsingle-output- (SISO-) based systems.

In network layer research using $\mathrm{CT}$, the nodes in the centralized cooperative cluster are often assumed to be colocated [12-15]; in this model, the disparate path losses caused by the different distances between the transmitting nodes in a cluster to the receiver node are ignored. The authors in [18] realized through simulation of some specific topologies that there can be a significant error (i.e., SNR penalty) incurred for making the colocated assumption.

Motivated by this fact, in this paper, we focus on the error in the colocated assumption, for the centralized cluster architecture with finite node degree. To be specific, we analyze the impact of path-loss disparity, which is ignored in the colocated approximation. When relays are randomly located around a cluster head, their disparate path losses cause variation in the statistics of received SNR or power at the receiver of the VMISO link compared to the colocated (or real) MISO link. Even though the centralized CT is highly compatible with the classical SISO-based system, this SNR variation 
ignored in the colocated assumption limits accurate design and evaluation of high-layer protocols based on CT. For this reason, we quantify the error of the colocated assumption in the presence of path-loss disparity by comparing the required SNRs to achieve a certain outage probability.

Beaulieu and $\mathrm{Hu}$ [19] derived a closed form expression for the outage probability at the destination for the case of the decode-and-forward (DF) relays, where the location of the relays is assumed to be known. The paper [19] has an intermediate result, where the number of relays that successfully decode is assumed to be known, while the final result in [19] allows for the opportunistic case, in which the number of relays that successfully decode is not known a priori. However, this final expression in [19] is long, complicated, and numerically sensitive [20]. Moreover, the SNR penalty for the colocated assumption is not considered in [19], nor are random locations of nodes taken into account.

In this paper, we allow the node locations to be random and analyze two-hop DF cooperative networks with multiple relays. Cho et al. [21] compared outage performances of random and best relay selection schemes assuming random relay locations, but they only assumed single-relay amplify-and-forward (AF) system. Wang et al. [22] also analyzed outage probabilities with the single-relay DF twohop network, where the relay is selected by different criteria. Zhai et al. [23] proposed a cooperative automatic repeat request (ARQ) scheme, in which randomly located relays try to retransmit in response to a negative acknowledgement (NACK) from the destination. However, if more than one relay accesses the channel simultaneously, collision occurs and the retransmission is unsuccessful. Aalo et al. in [24, 25] analyzed outage and average bit error rate (ABER) of multihop AF relay networks with randomly located nodes. However, it only assumed a single-relay node for each hop in the interference-limited scenario focusing on signal-tointerference ratio (SIR) instead of SNR.

On the other hand, Wang et al. [26] derived the outage rates for both single- and multirelay scenarios with a two-hop DF network. The authors assumed transmit power control for different nodes and maximum ratio transmission (MRT) [27], which is not practical because channel state information (CSI) should be known at both the transmitters (i.e., source and relays) and receiver (i.e., destination). Moreover, for some types of networks such as sensor networks, constraints on node (e.g., transmit power, processor, and memory) may preclude optimal power control. Also, the presence of multiple time and frequency offsets in CT may make channel estimation very challenging [28], especially when a large number of nodes do CT as in [3]. For the same reason, in this type of network, distributed beamforming schemes in $[29,30]$, which require CSI at the transmitters, are also difficult to implement in practice.

Therefore, we assume equal transmit power for any transmitting nodes with cooperative DF relaying, which does not require CSI at the transmitters. Moreover, while [26] provides an approximate outage rate, which is complex to interpret, we capture the impact of the random relay locations in an intuitive form. To be specific, for the known number of successfully decoding relays, we show that the random locations of nodes produce a random received power that, when averaged over multipath fading, has a log-normal distribution as in shadowing, when averaged over multipath fading. We also treat the opportunistic case and derive the outage performance for the case when the number of relays that successfully decode is not known a priori by assuming that the first-hop errors are independent and identically distributed (i.i.d.). The conference version of this paper [31] presents that a SNR penalty of up to $3 \mathrm{~dB}$ should be included, when making colocated assumption to achieve a certain target outage probability. This paper extends the analysis in [31] with a single approximate outage probability $\widetilde{P}_{\text {out }}$ based on the log-normal approximation, which is more accurate than the upper and lower bounds in [31]. Moreover, the log-normal distribution model of the path-loss disparity, which is the key contribution of this paper, is analyzed in more detail by the statistical normality test based on the Kolmogorov-Smirnov method. In other words, we evaluate the statistical validity of the log-normal model with different system parameters.

The organization of the paper is as follows. In Section 2, the system models including network topology, channel, and outage are presented. The following three sections correspond to three stages for the outage capacity approximation. In Section 3, first-hop error is approximated to decouple the first and second-hop errors. Then, the log-normal distribution to model the path-loss disparity in the second hop is presented in Section 4. Also, the equivalent log-normal model is evaluated by the cumulative distribution function (CDF) comparison and the statistical normality test. Section 5 is the final stage to derive the outage approximation in a closed form, in which first and second moments of channel gain parameter $\lambda$ are derived under the random relay locations. Section 6 provides the simulation results that show the error of the colocated assumption and the accuracy of the proposed approximate outage capacity $\widetilde{P}_{\text {out }}$.

\section{System Model}

We consider a VMISO communication in each hop consisting of two phases as shown in Figure 1, where the source, which is indicated by the left black dot at the center of the dotted lined circle, first transmits a packet to the destination (the right black dot) in Phase 1. After that, the multiple relays (the white-filled circles) around the source decode and then forward using orthogonal channels to the destination in Phase 2 [19]. We note that, instead of orthogonal transmission, space-time block codes (STBCs) can be used, in which case the analysis in this paper is still applicable with a slight modification. This cluster architecture is referred to as the centralized cluster [17], where each cluster has a cluster head that recruits its cooperating relays and triggers the group transmission.

2.1. Network Topology. We consider a static VMISO network as shown in Figure 2, where the source node is located at the origin with a distance $d_{0}$ to the destination. Also, there are $N$ number of cooperating relays around the source, which are 


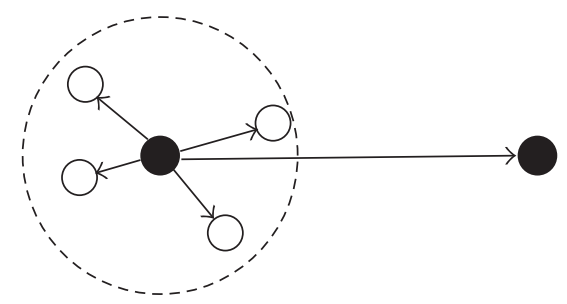

Phase 1: source transmission

(a)

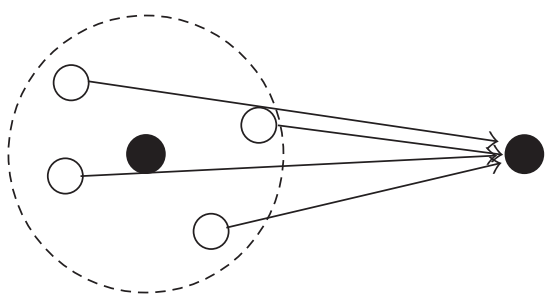

Phase 2: relay transmission

(b)

FIGURE 1: Two-hop cooperative transmission scenario.

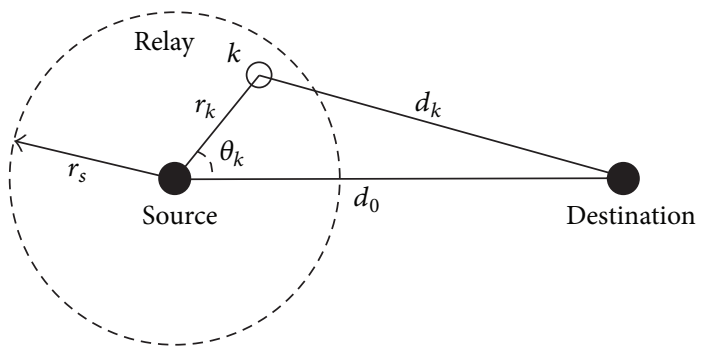

Figure 2: Network topology model.

located in the dotted lined circle with a radius $r_{s}$ centered at the source node. $r_{s}$ can be regarded as the SISO range, over which the source collects its cooperators. As in [12-14], we assume the "CT range extension case," where $1.5 \leq d_{0} / r_{s} \leq 4$, which was demonstrated in [32]. We note that maximum range extension happens when radios do not decrease their transmit powers when cooperating and they transmit using either orthogonal channels or space-time block coding, so the link benefits from diversity gain [20]. The colocated VMISO model in [12-14] assumes that the links between the source and the relays are error-free, which is another factor that causes the error of the colocated assumption.

We assume that $N$ relays are independently and identically distributed in the circle with the radius $r_{s}$ following the uniform distribution. As shown in Figure 2, relay $k$ represented by the white-filled circle exists at a distance of $r_{k}$ from the source with an angle $\theta_{k}$ with respect to the line connecting the source and destination. Also, relay $k$ is $d_{k}$ away from the destination, which determines the path loss between the relay and the destination. It follows that the polar coordinates $\left(r_{k}, \theta_{k}\right)$ of relay $k$ have the probability distribution functions (PDFs):

$$
\begin{aligned}
& f_{r_{k}}\left(r_{k}\right)=\frac{2 r_{k}}{r_{s}^{2}}, \\
& f_{\theta_{k}}\left(\theta_{k}\right)=\frac{1}{2 \pi},
\end{aligned}
$$

where $0<r_{k} \leq r_{s}$ and $0 \leq \theta_{k} \leq 2 \pi$, respectively.

2.2. Channel Model. We assume mutually independent Rayleigh fading for any links between two nodes (the source, relays, and destination). The node indices of the source and destination are zero and $N+1$, and the relay index $k$ satisfies $1 \leq k \leq N$. The complex channel gains are denoted by $h_{0: k}$ (from the source to relay $k$ ), $h_{0: N+1}$ (from the source to the destination), and $h_{k: N+1}$ (from relay $k$ to the destination). Hence, $\Omega_{i: j}=\left|h_{i: j}\right|^{2}$ follows the exponential distribution with a parameter $\lambda_{i: j}$ that is determined by the locations of nodes $i$ and $j$. Therefore, when the node locations are given, the cumulative distribution function (CDF) of $\Omega_{k}$ given $\lambda_{i: j}$ is expressed as

$$
F_{\Omega_{i: j} \mid \lambda_{i: j}}(x)=1-e^{-\lambda_{i: j} x}
$$

where the conditional mean and variance are $\mathbb{E}\left\{\Omega_{i: j} \mid \lambda_{i: j}\right\}=$ $1 / \lambda_{i: j}$ and $\mathbb{V A R}\left\{\Omega_{i: j} \mid \lambda_{i: j}\right\}=1 / \lambda_{i: j}^{2}$, respectively. If the pathloss exponent is $\alpha$, then $\lambda_{0: k}=r_{k}^{\alpha}$ for the source-relay link and $\lambda_{k: N+1}=d_{k}^{\alpha}$ for the relay-destination link.

2.3. Outage Model. In this section, we first consider the outage probability for a deterministic network topology (i.e., the relay locations are given), the closed form of which is provided in [19]. Next, we consider the random spatial distribution of the relays to capture how random path-loss disparities impact outage probability.

Assuming the same transmission powers for the source and $N$ relays, the conditional mutual information between the source and relay $k$ given that $\lambda_{0: k}=r_{k}^{\alpha}$ is

$$
\mathbf{I}_{0: k}\left(\operatorname{SNR} \mid \lambda_{0: k}\right)=\frac{1}{N+1} \log _{2}\left(1+\operatorname{SNR} \cdot \Omega_{0: k}\right),
$$

where SNR is the transmit SNR of each node [19]. Also, the normalization factor $1 /(N+1)$ is for orthogonal DF transmissions of the source and relays, where the destination achieves diversity gain. Therefore, for a certain transmission rate $\mathrm{R}$ (bit/Hz/sec), the probability that relay $k$ fails to decode the received signal from the source is given by

$$
p_{k}=F_{\Omega_{0: k} \mid \lambda_{0: k}}(g(\mathrm{SNR}, \mathrm{R}))=1-e^{-\lambda_{0: k} \cdot g(\mathrm{SNR}, \mathrm{R})},
$$

where $F_{\Omega_{0: k} \mid \lambda_{0: k}}(x)$ is the conditional CDF of $\Omega_{0: k}$, given $\lambda_{0: k}=$ $r_{k}^{\alpha}$, and $g(t, \mathrm{R})=\left(2^{(N+1) \mathrm{R}}-1\right) / t$.

Suppose that, for a given network topology, $\mathcal{S}$ is a particular set of $M$ relays that successfully decode the source transmission, where $0 \leq M \leq N$. Therefore, the conditional 
mutual information of the VMISO link, conditioned on $\mathcal{S}$, is given by

$$
\begin{aligned}
& \mathbf{I}(\operatorname{SNR} \mid \mathcal{S}) \\
& \quad=\frac{1}{N+1} \log _{2}\left(1+\operatorname{SNR} \cdot \Omega_{0: N+1}+\sum_{k \in \mathcal{S}} \operatorname{SNR} \cdot \Omega_{k: N+1}\right) .
\end{aligned}
$$

Thus, the outage probability of the VMISO communication for a given network topology indicated by two parameter vectors $\bar{\Lambda}_{s}=\left[\lambda_{0: 1}, \lambda_{0: 2}, \ldots, \lambda_{0: N}\right]^{T}$ and $\bar{\Lambda}_{d}=$ $\left[\lambda_{1: N+1}, \lambda_{2: N+1}, \ldots, \lambda_{N: N+1}\right]^{T}$ that satisfy $\lambda_{0: k}=r_{k}^{\alpha}$ and $\lambda_{k: N+1}=d_{k}^{\alpha}$, with $1 \leq \dot{k} \leq N$ is expressed as

$$
P_{\text {out } \mid \overline{\mathbf{\Lambda}}_{s}, \overline{\mathbf{\Lambda}}_{d}}(\mathrm{SNR}, \mathrm{R})=\sum_{\mathcal{S}} \operatorname{Pr}[\mathbf{I}<\mathrm{R} \mid \mathcal{S}] \cdot \operatorname{Pr}[\mathcal{S}],
$$

where $\operatorname{Pr}[\mathcal{S}]=\left(\prod_{k \notin \mathcal{S}} p_{k}\right) \cdot\left(\prod_{k \in \mathcal{S}}\left(1-p_{k}\right)\right)$. This equation is the same as in [19] except that we suppose $\operatorname{Pr}[\mathcal{S}]$ is the probability conditioned on the certain network topology (i.e., $\bar{\Lambda}_{s}$ and $\bar{\Lambda}_{d}$ ). Considering the randomness of the relay locations, the outage rate averaged over the random network topology has $N^{2}$-fold integral as

$$
\begin{aligned}
P_{\text {out }}(\operatorname{SNR}, \mathrm{R})=\int \cdots \int & P_{\text {out } \mid \bar{\Lambda}_{s}, \bar{\Lambda}_{d}}(\operatorname{SNR}, \mathrm{R}) \\
\times & \prod_{k=1}^{N}\left[f_{\lambda_{0: k}, \lambda_{k: N+1}}\left(\lambda_{0: k}, \lambda_{k: N+1}\right)\right. \\
& \left.\times d \lambda_{0: k} d \lambda_{k: N+1}\right],
\end{aligned}
$$

where $f_{\lambda_{0: k}, \lambda_{k: N+1}}\left(x_{1}, x_{2}\right)$ is the joint PDF of $\lambda_{0: k}$ and $\lambda_{k: N+1}$, which can be obtained by the variable transformation from $f_{r_{k}}\left(r_{k}\right)$ and $f_{\theta_{k}}\left(\theta_{k}\right)$.

\section{First-Hop Error Approximation}

It is difficult to obtain the closed form expression of the outage probability under the random relay locations in (8), because the first hop (between the source and the relays) and second hop (between the relays and the destination) depend on each other by the random relay locations. Therefore, to obtain a single estimate of the outage probability with random relay locations, we first approximate the first-hop error rates of the $N$ relays by independent Bernoulli trials. Then, assuming that $M$ out of $N$ relays successfully decode the first-hop transmission from the source, we derive the second-hop outage probability for a given (deterministic) relay topology.

3.1. Bernoulli Trial Approximation of First-Hop Error. The first-hop outage rate of relay $k$ in (5) satisfies that $0 \leq p_{k} \leq$ $p_{\max }=1-e^{-r_{s}^{\alpha} \cdot g(\mathrm{SNR}, \mathrm{R})}$, because $0<r_{k} \leq r_{s}$. To decouple the outage rates between the first (source-relays) and second (relays-destination) hops, we approximate the first-hop error rates of the $N$ relays by independent Bernoulli trials with the same probability $p$. If all $N$ relays have the same constant first-hop error rate of $p$, where $0 \leq p \leq p_{\max }, P_{\text {out } \mid \bar{\Lambda}_{s}, \bar{\Lambda}_{d}}$ in Section 2.3, which does not depend on $\bar{\Lambda}_{s}$ anymore, is simplified as

$$
\begin{aligned}
& P_{\text {out } \mid \overline{\mathbf{\Lambda}}_{d}}(\mathrm{SNR}, \mathrm{R}) \\
& =\sum_{M=0}^{N}\left(\begin{array}{l}
N \\
M
\end{array}\right) p^{N-M}(1-p)^{M} \operatorname{Pr}\left[\sum_{k=0}^{M} \Omega_{k: N+1}<g(\text { SNR, R })\right] .
\end{aligned}
$$

Also, if $Y=\sum_{k=0}^{M} \Omega_{k: N+1}$, then $\operatorname{Pr}\left[\sum_{k=0}^{M} \Omega_{k: N+1}<g(\right.$ SNR, R $\left.)\right]=$ $F_{Y \mid M, \bar{\Lambda}_{d(1: M)}}(g(\operatorname{SNR}, \mathrm{R}))$ that is the conditional CDF of $Y$ for given $M$ and a truncated vector with $M$ elements out of $N$, $\bar{\Lambda}_{d(1: M)}=\left[\lambda_{1: N+1}, \ldots, \lambda_{M: N+1}\right]^{T}$. Therefore, the final outage in (8) is approximated by $N$-fold integral by

$$
\begin{aligned}
& \widetilde{P}_{\text {out }}(\text { SNR, R }) \\
& =\sum_{M=0}^{N}\left(\begin{array}{l}
N \\
M
\end{array}\right) p^{N-M}(1-p)^{M} \\
& \quad \times \int \cdots \int F_{Y \mid M, \bar{\Lambda}_{d(1: M)}}(g(\operatorname{SNR}, \mathrm{R})) \\
& \quad \times f_{\lambda_{1: N+1}}\left(\lambda_{1: N+1}\right) \cdots f_{\lambda_{N: N+1}} \\
& \quad \times\left(\lambda_{N: N+1}\right) d \lambda_{1: N+1} \cdots d \lambda_{N: N+1} .
\end{aligned}
$$

The upper and lower bounds of outage capacity in [31] are obtained using $p=p_{\max }$ and $p=0$, respectively. Alternatively (i.e., new in this paper), to obtain a single estimate of the outage rate with higher accuracy than the two bounds, we will use the average first-hop outage rate $p=\mathbb{E}\left\{p_{k}\right\}$, which is derived by (1) and (5) as

$$
\begin{aligned}
p & =\mathbb{E}\left\{p_{k}\right\}=\int_{0}^{r_{s}}\left(1-e^{-\left(g(\mathrm{SNR}, \mathrm{R}) / x^{\alpha}\right)}\right) \cdot \frac{2 x}{r_{s}^{2}} d x \\
& =1+\frac{2}{\alpha} \cdot P_{\varepsilon}\left(1-\frac{-2}{\alpha}, g(\mathrm{SNR}, \mathrm{R}) \cdot r_{s}^{\alpha}\right),
\end{aligned}
$$

where $P_{\varepsilon}(n, z)$ is $n$th order exponential integral [33], which is given by

$$
P_{\varepsilon}(n, z)=\int_{1}^{\infty} \frac{\exp (-z t)}{t^{n}} d t
$$

We note that this exponential function can be easily calculated by popular numerical tools such as MATLAB, MATHEMATICA, and MAPLE [34].

3.2. Second-Hop Error and Path-Loss Disparity. In this section, we look at the second-hop outage rate $P_{\text {out } \mid \bar{\Lambda}_{d}}(\mathrm{SNR}, \mathrm{R})$ for a given relay topology, assuming the number of active relays participating in the second-hop transmission is $M$. As a general case with path-loss disparities, when $\lambda_{k: N+1} \neq \lambda_{j: N+1}$ for $k \neq j, F_{Y \mid M, \bar{\Lambda}_{d(1: M)}}(y)$ in (10) follows the hypoexponential distribution as

$$
F_{Y \mid M, \bar{\Lambda}_{d(1: M)}}(y)=1-\sum_{k=0}^{M} A_{k} \exp \left(-\lambda_{k} y\right),
$$

where $A_{k}=\prod_{k \neq j}\left(\lambda_{j} /\left(\lambda_{j}-\lambda_{k}\right)\right)$ and $\sum_{i=0}^{M} A_{k}=1[20]$. 
On the other hand, when $\lambda_{0: N+1}=\lambda_{1: N+1}=\cdots=$ $\lambda_{M: N+1}=\lambda$, which corresponds to the colocated antenna array with $M$ elements, $F_{Y \mid M, \bar{\Lambda}_{d(1: M)}}(y)$ is the CDF of the gamma distribution as

$$
F_{Y \mid M, \lambda}(y)=1-\sum_{k=0}^{M} \frac{\exp (-\lambda y) \cdot(\lambda y)^{k}}{k !} .
$$

Therefore, as shown in the equation, $F_{Y \mid M, \bar{\Lambda}_{d(1: M)}}(y)$ can be simplified into $F_{Y \mid M, \lambda}(y)$ with the single condition variable $\lambda$ instead of the condition vector $\bar{\Lambda}_{d(1: M)}$. Also, the outage probability in (10) can be expressed with a single integral as

$$
\begin{aligned}
\widetilde{P}_{\text {out }}(\text { SNR }, \mathrm{R}) \\
=\sum_{M=0}^{N}\left(\begin{array}{l}
N \\
M
\end{array}\right) p^{N-M}(1-p)^{M} \\
\quad \times \int F_{Y \mid M, \lambda}(g(\operatorname{SNR}, \mathrm{R})) f_{\lambda \mid M}(\lambda) d \lambda .
\end{aligned}
$$

We note that, for the colocated assumption, $P_{\text {out }}=$ $F_{Y \mid M=N, \lambda=d_{0}^{\alpha}}(g(\operatorname{SNR}, \mathrm{R}))$ without any integral, because all the links have the same distances of $d_{0}$ to the destination. Compared to (10), the outage equation in (15) is much simpler, because there is only one integral. Also, the conditional CDF $F_{Y \mid M, \overline{\mathbf{\Lambda}}_{d(1: M)}}(y)$ in (13) is numerically sensitive to compute [20]. Therefore, ultimately to capture the impact of pathloss disparity, we use an approximation to project the vector $\bar{\Lambda}_{d(1: M)}=\left[\lambda_{1: N+1}, \ldots, \lambda_{M: N+1}\right]^{T}$ into a single variable $\lambda$ assuming high SNR, while keeping the influence of the random relay locations on the outage rate, in the following section.

\section{Path-Loss Disparity Modeling by Log-Normal Distribution}

In this section, assuming high SNR, we will first approximate the hypoexponential distribution in (13) using the gamma distribution in (14), because the hypoexponential distribution is long, complicated, and numerically sensitive [20]. Also, the approximation into the gamma distribution enables us to characterize the path-loss disparity as the log-normal distribution, which means the impact of the path-loss disparity on the outage rate can be captured by the equivalent log-normal shadowing. Therefore, the final outage rate formula derived in this section is the same as the outage capacity in the conventional composite channel with Rayleigh multipath fading and log-normal shadowing. This log-normal approximation is validated by the illustrative $\mathrm{CDF}$ comparison and the statistical normality test using the Kolmogorov-Smirnov (KS) method.

4.1. Gamma Approximation of $F_{Y \mid M, \overline{\boldsymbol{\Lambda}}_{d(1: M)}}(y)$ into $F_{Y \mid M, \lambda}(y)$. Many studies on CT focus on the asymptotic performance by the limit of SNR $\rightarrow \infty$ [20]. In [20], the author proposes a simple but accurate way to calculate outage performance of
CT with dissimilar (deterministic) path losses, where the outage probability based on hypoexponential distribution in (13) can be approximated by the outage probability computation using gamma distribution in (14) with a negligible error. This enables us to use the traditional notion of the performance analysis based on the real multiple-antenna array system to the VMISO link. The key result in this approximation in [20] is

$$
\lambda \approx\left(\prod_{k=0}^{M} \lambda_{k: N+1}\right)^{1 /(M+1)} .
$$

In other words, in this gamma approximation, the single parameter $\lambda$ is equal to the geometric mean of the distinct $\lambda_{k: N+1}$ for $k=0,1, \ldots, M$. Please see the appendix for the proof, which is relatively simpler than [20].

4.2. Approximation of $f_{\lambda \mid M}(x)$ by Log-Normal Distribution. To calculate the outage rate with the random relay locations in (15) with the gamma approximation, we need to obtain the conditional PDF of $\lambda$ for given $M$. In $\mathrm{dB},(16)$ is expressed as

$$
10 \log _{10} \lambda \approx \frac{10}{M+1} \sum_{k=0}^{M} \log _{10} \lambda_{k: N+1}
$$

where $\lambda_{0: N+1}=d_{0}^{\alpha}$ is deterministic, while $\lambda_{1: N+1}, \ldots, \lambda_{M: N+1}$ are i.i.d. random variables, when $d_{0}, r_{s}$, and $\alpha$ are given.

Because (17) is the sum of i.i.d variables, we model $10 \log _{10} \lambda$ as a Gaussian distribution. In other words, $\lambda$ is approximated by log-normal distribution, which becomes more accurate, as $M$ increases by the Central Limit Theorem (CLT) [35]. The log-normal approximation is also convenient, because it enables us to use the conventional composite channel model (Rayleigh multipath fading and log-normal shadowing). By the log-normal approximation, we have the PDF $f_{\lambda \mid M}(x)$ as below:

$$
f_{\lambda \mid M}(x) \approx \frac{1}{x \xi \sqrt{2 \pi} \sigma} \exp \left[-\frac{\left(10 \log _{10} x-\mu\right)^{2}}{2 \sigma^{2}}\right],
$$

where $0<x<\infty, \xi=\ln 10 / 10$. Also, because $\lambda_{k: N+1}=d_{k}^{\alpha}$,

$$
\begin{gathered}
\mu=\mathbb{E}\left\{\lambda_{(\mathrm{dB})} \mid M\right\}=\frac{10 \alpha}{M+1}\left(\log _{10} d_{0}+M \mathbb{E}\left\{\log _{10} d_{k}\right\}\right), \\
\sigma^{2}=\mathbb{V} \mathbb{A} \mathbb{R}\left\{\lambda_{(\mathrm{dB})} \mid M\right\}=\frac{100 \alpha^{2} M}{(M+1)^{2}} \mathbb{V A \mathbb { R }}\left\{\log _{10} d_{k}\right\},
\end{gathered}
$$

where $\mathbb{E}\left\{\log _{10} d_{k}\right\}$ and $\mathbb{V} \mathbb{R}\left\{\log _{10} d_{k}\right\}$ are derived in Section 5, which presents the distribution of $d_{k}$.

Using this log-normal approximation, the influence of the random relay locations can be characterized by the lognormal approximation of the PDF of $\lambda$, which means that the random separations have the same effect as log-normal shadowing on the outage. Based on this approximation, 
the outage probability $\widetilde{P}_{\text {out }}$ in (15) can be simplified in a summation form using the Gauss-Hermite method [36]:

$$
\begin{aligned}
& \widetilde{P}_{\text {out }}(\text { SNR }, \mathrm{R}) \\
& \quad \approx \sum_{M=0}^{N}\left(\begin{array}{l}
N \\
M
\end{array}\right) \frac{p^{N-M}(1-p)^{M}}{\sqrt{\pi}}\left[\sum_{i=1}^{m} W_{i} \cdot G\left(10^{\left(\sqrt{2} \sigma r_{i}+\mu\right) / 10}\right)\right],
\end{aligned}
$$

where $G(x)=F_{Y \mid M, \lambda=x}(g(\operatorname{SNR}, \mathrm{R})), W_{i}$ are weight factors, $r_{i}$ are the roots of the Hermite polynomial, and $m$ is the order of the Hermite polynomial. We test this log-normal approximation in the following section.

4.3. Log-Normal Approximation Test. In this section, we test the quality of the log-normal approximation in (18) with different system parameters. First, we compare empirical CDF of $\lambda_{\mathrm{dB}}=10 \log _{10} \lambda$ in (17) and its normal CDF fit by matching their mean and variance.

(1) CDF Comparison Example. Figure 3 shows three CDFs of $\lambda$ in $\mathrm{dB}, F_{\lambda_{(\mathrm{dB})} \mid M}(x)$, when $\alpha=4$ and $r_{s}=1$. The results in the three subfigures are achieved with different distances from the source to destination $d_{0}$ and the number of active relays $M$. To be specific, Figures 3(a), 3(b), and 3(c) correspond to $\left(d_{0}, M\right)=(3,4),(3,1)$, and $(1.5,4)$, respectively. In each figure, the red solid curve indicates the real empirical CDF $F_{\lambda_{(\mathrm{dB})} \mid M}(x)$ with $10^{4}$ samples, while the black dotted line with the "o"-markers represents the normal fit of $\lambda_{(\mathrm{dB})}$ (i.e., the lognormal fit of $\lambda$ ).

In Figure 3(a) obtained with $\left(d_{0}, M\right)=(3,4)$, the two CDF curves are almost identical, which means the lognormal approximation in (18) is highly accurate. On the other hand, when the number of active relays $M$ decreases to one as in Figure 3(b), the normal fit shows a significantly greater error from the empirical CDF compared to Figure 3(a). Also, with $d_{0}=1.5$ and $M=4$, Figure 3(c) shows a smaller error of the normal fit compared to Figure 3(b). On the other hand, compared to Figure 3(a) with $d_{0}=3$ and $M=4$, the error of the normal fit in Figure 3(c) is greater. Thus, comparing the three figures, we can conclude that the quality of the log-normal approximation in (18) becomes more accurate, as $M$ and $d_{0}$ increase. We note that the quality of the log-normal approximation does not depend on $\alpha$, because $10 \log _{10} \lambda_{k: N+1}=10 \alpha \log _{10} d_{k: N+1}$, where $\alpha$ is a product term.

However, the outage probability is determined by the tail property of the channel distribution, and the log-normal approximation has a longer tail than the original conditional PDF $f_{\lambda \mid M}(x)$, especially when $M$ is small. Therefore, this outage rate based on the log-normal approximation is higher than (15), which is used to derive the upper bound of the outage rate in the following section.

(2) Statistical Normality Test: Kolmogorov-Smirnov. In this section, we use a well-known statistical normality test: the KS tests [37] at significance level 5\%. The KS method is widely used to assess a null hypothesis that distribution of the data is normal in various applications [38-40]. We note
TABLE 1: KS test $P$ value results $\left(r_{s}=1, \alpha=4\right)$.

\begin{tabular}{ccccc}
\hline$d_{0}$ & \multicolumn{4}{c}{$M$} \\
& 1 & 2 & 4 & 8 \\
\hline 1.5 & $4.777 \times 10^{-6}$ & $1.221 \times 10^{-2}$ & 0.1479 & 0.3566 \\
2.0 & $1.257 \times 10^{-4}$ & $7.352 \times 10^{-2}$ & 0.3230 & 0.5228 \\
2.5 & $6.162 \times 10^{-4}$ & 0.1539 & 0.4360 & 0.6088 \\
3.0 & $1.531 \times 10^{-3}$ & 0.2279 & 0.5055 & 0.6558 \\
3.5 & $2.593 \times 10^{-3}$ & 0.2855 & 0.5577 & 0.6858 \\
4.0 & $3.763 \times 10^{-3}$ & 0.3265 & 0.5964 & 0.7012 \\
\hline
\end{tabular}

that the KS test tends to be more sensitive near the center of the distribution than at the tails. However, [41] shows that the KS method is as powerful as other tests (e.g., ShapiroWilk, Anderson-Darling, and Lilliefors), when the number of samples is large enough (more than or equal to 1,000 samples).

We perform the KS test using 2,000 samples with different $d_{0}$ and $M$, when $r_{s}=1$ and $\alpha=4$. Table 1 shows the $P$ values averaged over $10^{6}$ iterative tests. The six rows correspond to $d_{0}$ from 1.5 to 4.0 with the same (0.5) interval, while the four columns represent $M=1,2,4$, and 8 , respectively. In the table, the $P$ value increases, as $d_{0}$ and $M$ increase.

Figure 4 shows the corresponding probability of passing test, which is averaged over $10^{6} \mathrm{KS}$ tests, with the significance level of 5\%. As in Table 1, we use $\alpha=4$ and $r_{s}=1$. In the figure, the horizontal axis indicates the distance between the source and destination $1.5 \geq d_{0} \geq 4$. On the other hand, the vertical axis indicates test-passing probability, which means the probability that $P$ value is greater than the $5 \%$ significance level over $10^{6}$ iterative KS tests. Also, the blue solid line, the red dashed line, and the black dotted line represent $M=2$, 4 , and 8 , respectively. This figure shows the same trends as in Table 1 with respect to $d_{0}$ and $M$; it becomes more likely to pass the test, as $d_{0}$ and $M$ increase. In other words, the log-normal approximation in (18) becomes a better fit, as $d_{0}$ and $M$ increase. Moreover, when $d_{0} \geq 2$ and $M \geq 4$, the probability of passing is greater than $90 \%$, which means the log-normal fit is good.

\section{Distance to Destination under Random Relay Locations}

To derive the approximated outage probability in the previous section, the disparate path losses, which depend on the distances to destination $d_{k}$, need to be calculated. In particular, to obtain the complete outage rate in (20), $\mathbb{E}\left\{\log _{10} d_{k}\right\}$ and $\mathbb{V A R}\left\{\log _{10} d_{k}\right\}$ should be obtained for $\mu$ and $\sigma^{2}$. In this section, we first derive the PDF of $d_{k}, f_{d_{k}}(x)$, which characterizes the distance from relay $k$ to the destination. However, because it is difficult to obtain closed form solutions of $\mathbb{E}\left\{\log _{10} d_{k}\right\}$ and $\mathbb{V A R}\left\{\log _{10} d_{k}\right\}$, therefore, as the final stage to derive an approximate outage rate in the presence of the path-loss disparity caused by the random relay locations, we approximate $f_{d_{k}}(x)$ and derive the corresponding $\mu$ and $\sigma^{2}$ in closed forms. 


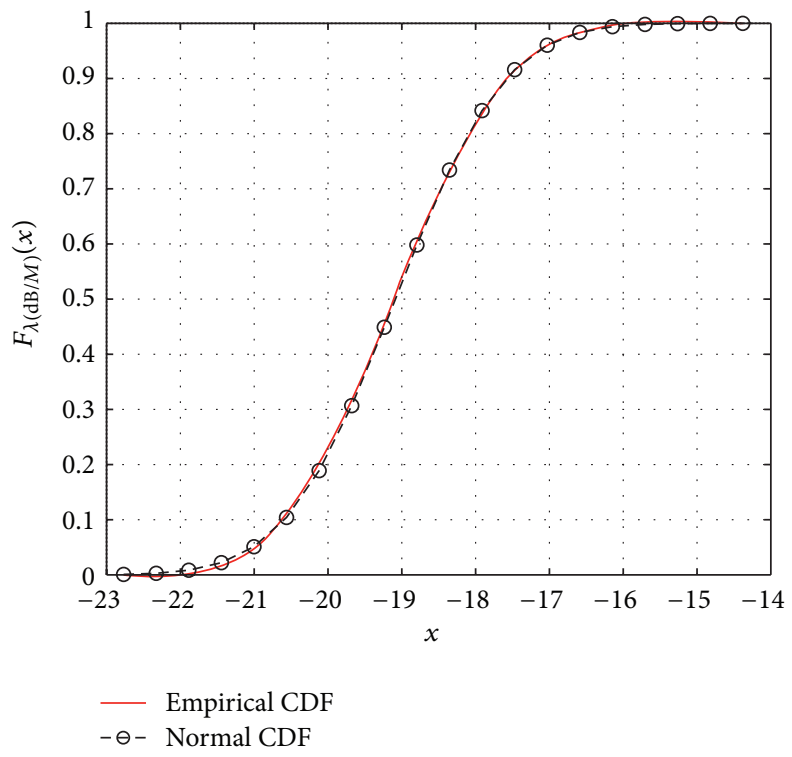

(a) $d_{0}=3$ and $M=4$

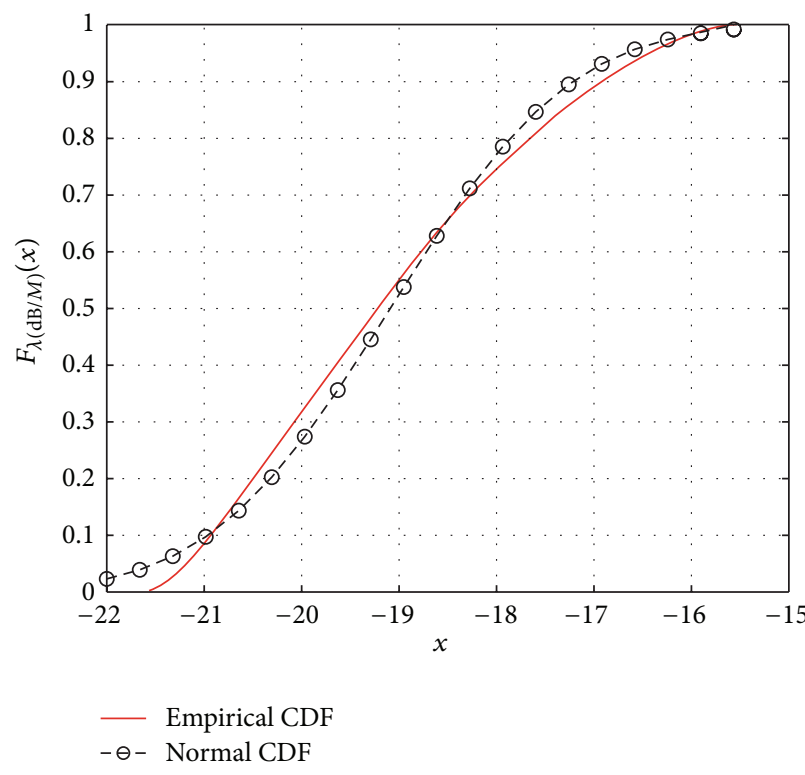

(b) $d_{0}=3$ and $M=1$

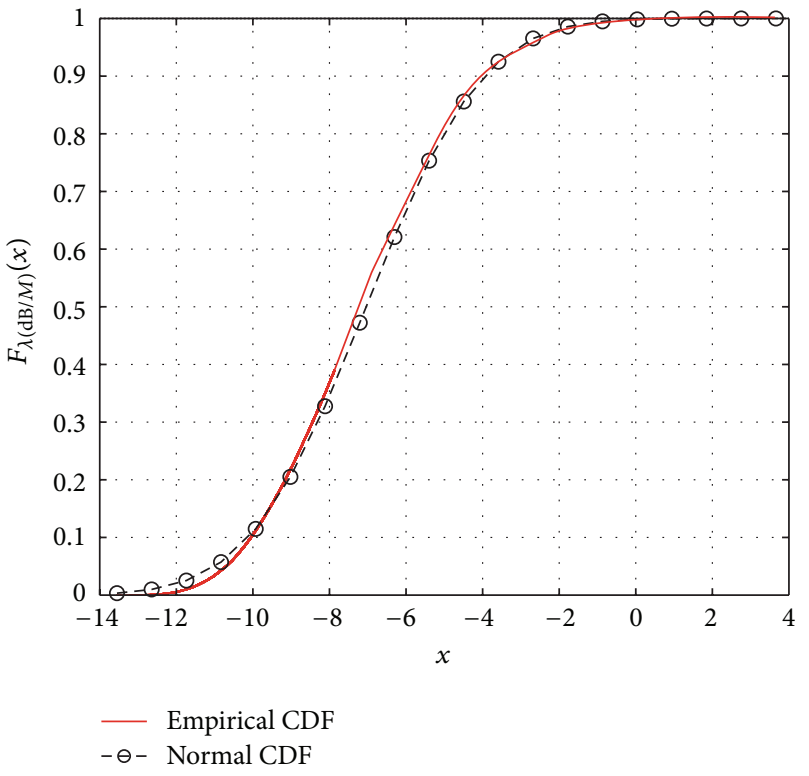

(c) $d_{0}=1.5$ and $M=4$

FIGURE 3: Normal CDFs compared with the empirical CDFs, $F_{\lambda_{(\mathrm{dB})} \mid M}(x)$, when $\alpha=4$ and $r_{s}=1$.

5.1. Original Probability Distributions of $d_{k}$. Figure 5 illustrates the variable assignments, which are used to derive the PDF of $d_{k}$, where a relay node is randomly located inside the left circle centered at the origin $O$, where the source exists. Also, the destination node is at the distance $d_{0}$ from the origin on the $x$-axis, and the relay node is $d_{k}$ away from the destination. The locations $A, B, B^{\prime}$, and $C$ indicate some important intersection points that will be used in the derivation of the probability distribution. Using the illustration, the CDF of $d_{k}$ can be the ratio of the area of the shaded region $(S)$ to the total area of the left circle, which is given by

$$
F_{d_{k}}(x)=\frac{S}{\pi r_{s}^{2}}
$$

where $d_{0}-r_{s} \leq x \leq d_{0}+r_{s}$, and the area $S$ can be expressed as

$$
S=\theta_{k} r_{s}^{2}+\phi_{k} x^{2}-r_{s} d_{0} \sin \theta_{k}
$$




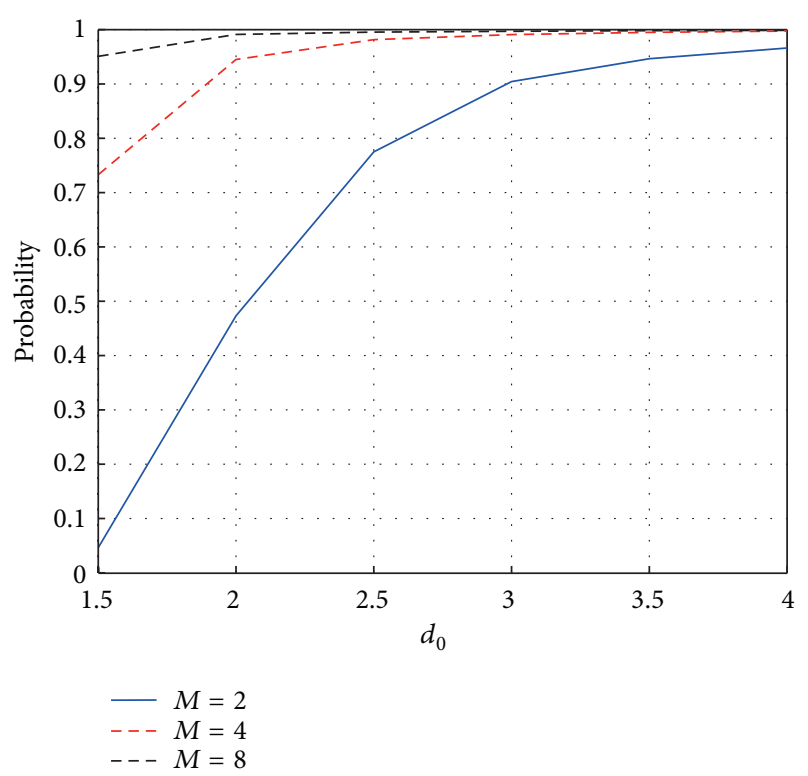

FIgURE 4: Probability of passing tests with $r_{s}=1$ and $\alpha=4$.

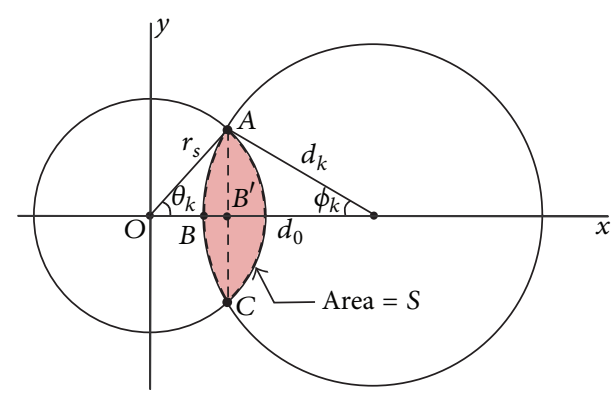

FIGURE 5: Illustration for derivation of PDF of $d_{k}$.

where $\theta_{k}=\arccos \left(\left(r_{s}^{2}+d_{0}^{2}-x^{2}\right) / 2 r_{s} d_{0}\right), \phi_{k}=\arccos \left(\left(x^{2}+\right.\right.$ $\left.d_{0}^{2}-r_{s}^{2}\right) / 2 x d_{0}$ ). Hence, the PDF of $d_{k}$ is given by

$$
\begin{aligned}
f_{d_{k}}(x)=\frac{1}{\pi r_{s}^{2}} \frac{\partial S}{\partial x} & \\
=\frac{x}{\pi r_{s}^{2}}( & \frac{r_{s} / d_{0}}{\sqrt{1-\left(\left(d_{0}^{2}-x^{2}+r_{s}^{2}\right)^{2} / 4 d_{0}^{2} r_{s}^{2}\right)}} \\
& -\frac{\left(\left(x / d_{0}\right)-\left(\left(d_{0}^{2}+x^{2}-r_{s}^{2}\right) / 2 d_{0} x\right)\right)}{\sqrt{1-\left(\left(d_{0}^{2}+x^{2}-r_{s}^{2}\right)^{2} / 4 d_{0}^{2} x^{2}\right)}} \\
& -\frac{\left(d_{0}^{2}-x^{2}+r_{s}^{2}\right) / 2 d_{0} r_{s}}{\sqrt{1-\left(\left(d_{0}^{2}-x^{2}+r_{s}^{2}\right)^{2} / 4 d_{0}^{2} r_{s}^{2}\right)}} \\
& \left.+2 \arccos \left(\frac{d_{0}^{2}+x^{2}-r_{s}^{2}}{2 d_{0} x}\right)\right)
\end{aligned}
$$

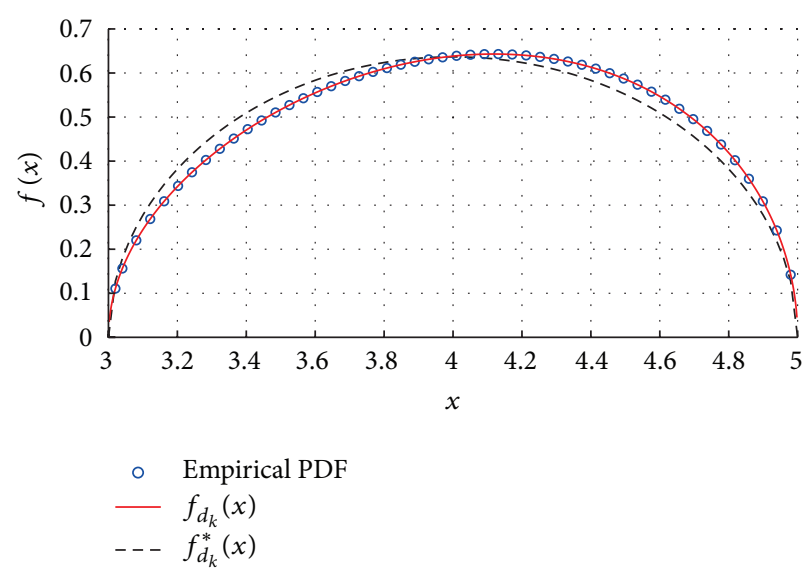

FIGURE 6: PDF of $d_{k}$ and its approximations, when $r_{s}=1$ and $d_{0}=4$.

where $\partial S / \partial d_{k}$ is the length of the arc $\overline{A B C}$ in Figure 5. However, this original PDF of $d_{k}$ does not give the closed form expressions of its mean and variance.

5.2. Approximation of $f_{d_{k}}(x)$. For the lack of the closed form expressions of the two statistics, we propose an approximated PDF of $d_{k}$ by using the chord $\overline{A B^{\prime} C}$, which is indicated by the dotted straight line in Figure 5, instead of the $\operatorname{arc} \overline{A B C}$. When $d_{0}$ is large enough compared to $r_{s}$ in Figure 2 (i.e., the $\operatorname{arc} \overline{A B C}$ and chord $\overline{A B^{\prime} C}$ are almost the same), the original PDF of $d_{k}$, $f_{d_{k}}(x)$ can be simplified into

$$
f_{d_{k}}^{*}(x)=\frac{2}{\pi r_{s}^{2}} \sqrt{r_{s}^{2}-\left(d_{0}-x\right)^{2}},
$$

where $d_{0}-r_{s} \leq x \leq d_{0}+r_{s}$.

As an example, in Figure 6, when $d_{0}=4$ and $r_{s}=1$, the empirical PDF, obtained with $5 \times 10^{6}$ random samples, is indicated by the blue "o"-markers. Also, the original PDF $f_{d_{k}}(x)$ is represented by the black solid line, where $f_{d_{k}}^{*}(x)$ is indicated by the black dotted line. As shown in the figure, the empirical PDF is identical to $f_{d_{k}}(x)$ in (23), which verifies its derivation. Moreover, $f_{d_{k}}^{*}(x) \approx f_{d_{k}}(x)$ in the domain $d_{0}-r_{s} \leq$ $x \leq d_{0}+r_{s}$, which confirms the validity of $f_{d_{k}}^{*}(x)$.

Moreover, this approximated PDF $f_{d_{k}}^{*}(x)$ has the mean and variance in closed forms as $\mathbb{E}\left\{d_{k}\right\}=d_{0}$ and $\mathbb{V A R}\left\{d_{k}\right\}=$ $r_{s}^{2} / 4$. Therefore, by the Taylor expansion, $\mathbb{E}\left\{\log _{10} d_{k}\right\} \approx$ $\log _{10} d_{0}$ and $\operatorname{VAR}\left\{\log _{10} d_{k}\right\} \approx r_{s}^{2} / 4\left(d_{0} \ln 10\right)^{2}$. Therefore, we can obtain $\mu$ and $\sigma^{2}$ as

$$
\begin{gathered}
\mu=10 \alpha \log _{10} d_{0}, \\
\sigma^{2}=\frac{25 M}{(M+1)^{2}}\left(\frac{\alpha r_{s}}{d_{0} \ln 10}\right)^{2} .
\end{gathered}
$$

By plugging these two parameters with the average first-hop probability $p=\mathbb{E}\{p\}$ in (11) into (20), the outage rate $\widetilde{P}_{\text {out }}$ in (20), which is approximated form of the real $P_{\text {out }}$ in (8), can be obtained. 


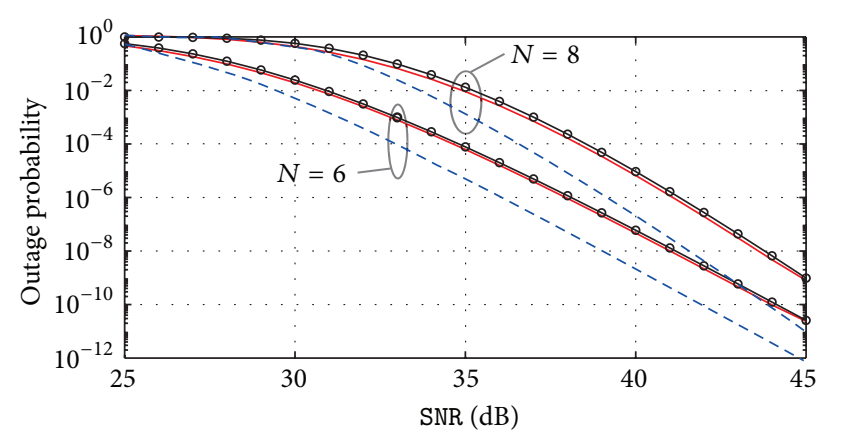

$$
\begin{aligned}
& --- \text { Colocated assumption } \\
& - \text { Monte Carlo (MC) } \\
& \rightarrow \widetilde{P}_{\text {out }} \text { (LN approximation) }
\end{aligned}
$$

Figure 7: Outage curves, when $\mathrm{R}=1, d_{0}=2, r_{s}=1, \alpha=4$, and $N=6,8$.

\section{Simulation Results}

In this section, we first compare the outage probability curves of three cases: the real outage obtained by Monte Carlo simulation, the colocated assumption outage, and the proposed estimate based on the three-stage approximations in the previous sections. Then, we measure the error of the colocated assumption by calculating an SNR gap to achieve a certain outage rate $10^{-6}$. Also, the SNR gap caused by the path-loss disparity is compared with the SNR gap calculated by the proposed outage rate approximation $\widetilde{P}_{\text {out }}$ in $(20)$ by the log-normal distribution.

6.1. Outage Probability. Figure 7 shows the outage simulation results with $\mathrm{R}=1, d_{0}=2, r_{s}=1$, and $\alpha=4$. In the figures, the horizontal axis is SNR in $\mathrm{dB}$, while the vertical axis indicates the outage probability. There are two groups of the curves: the group having the higher outage rates with the steeper slopes corresponds to $N=8$, while the lower group corresponds to $N=6$. We note that the heights of the $N=8$ group are greater than the other group with $N=6$ because we assume orthogonal channel transmissions of the relays. If space-time block codes are used, the outage curves of the two groups are parallel, with a translation distance corresponding to the code rate, which can be readily calculated.

The blue dashed lines represent the outage rates based on the colocated assumption that ignores the path-loss disparity and first-hop errors. Also, the red solid curves are the true outage probabilities obtained by the Monte Carlo simulation (MC), while the solid line with the "o"-markers indicates the proposed outage approximation $\widetilde{P}_{\text {out }}$ by log-normal (LN) approximation.

Therefore, the gaps between the blue dashed lines and the red solid lines are the errors caused by the colocated assumption. For example, when the target outage rate is $10^{-6}$, the errors are about 1.75 and $1.8 \mathrm{~dB}$ for $N=6$ and 8 , respectively. In other words, to achieve the outage probability of $10^{-6}$ considering the relay separations and the source-relay link errors, more transmission power (e.g., 1.75 and $1.8 \mathrm{~dB}$

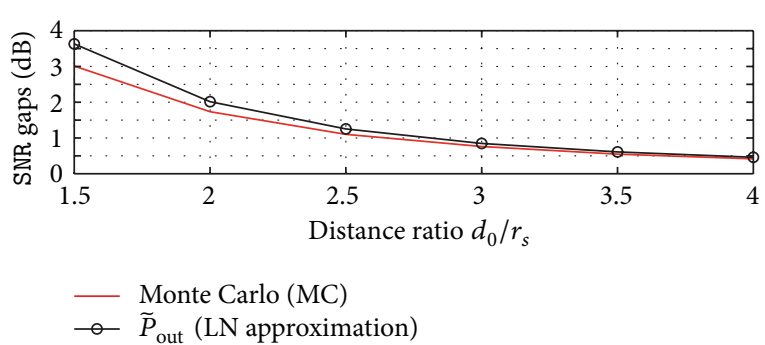

Figure 8: SNR gaps versus $d_{0} / r_{s}$, when $r_{s}=1, \alpha=4$, and $N=6$.

for $N=6$ and 8 , resp.) is required than is indicated by the colocated assumption.

Moreover, in the both groups, the real outage curves obtained by Monte Carlo simulation are very close to $\widetilde{P}_{\text {out }}$ in the high SNR regime (when the outage is less than $10^{-4}$ ), which implies the proposed approximation $\widetilde{P}_{\text {out }}$ has good accuracy. Therefore, the SNR penalty of the colocated assumption (i.e., the error in terms of SNR to achieve the target outage rate) can be estimated by $\widetilde{P}_{\text {out }}$ that characterizes the impact of the path-loss disparity by the equivalent lognormal shadowing model, following the conventional notion of the composite channel.

6.2. SNR Penalty Depending on System Parameters. In this section, we look at the error of the colocated assumption in terms of the SNR penalty or gap to achieve the outage probability of $10^{-6}$ depending on three system parameters: the distance ratio $d_{0} / r_{s}$, the path-loss exponent $\alpha$, and the number of relays $N$. In the simulation results shown in Figures 8, 9, and 10, there are two SNR gaps to the colocated assumption: the real error based on MC (the red solid lines) and the estimated errors by $\widetilde{P}_{\text {out }}$ with the log-normal (LN) approximation (the black curve with the "o"-markers, resp.). In the three figures, the real error is much closer to the error based on $\widetilde{P}_{\text {out }}$, compared to the upper and lower bounds in the conference paper version of this paper [31].

Figure 8 shows the SNR penalty in $\mathrm{dB}$ indicated by the $y$-axis for the different distance ratio $d_{0} / r_{s}$ represented by the $x$-axis, when $r_{s}=1, \alpha=4$, and $N=6$. The two SNR gaps decrease as $d_{0} / r_{s}$ increases, because the impact of the path-loss disparity becomes negligible for high $d_{0} / r_{s}$, which is also noticed by that the two variances $\sigma^{2}$ and $\sigma_{l}^{2}$ of the equivalent log-normal shadowing model are decreasing functions of $d_{0} / r_{s}$. Also, the first-hop error ignored in the colocated assumption is relatively much smaller than the second-hop error, when $d_{0} / r_{s}$ is large. However, considering that $d_{0} / r_{s}=1.5$ and 2 are the CT range extension ratios widely assumed in the VMISO-based protocol studies, the corresponding SNR gaps are large enough to degrade the protocols designed and operated with the colocated assumption. Moreover, if comparing the two SNR gap curves, the proposed approximation $\widetilde{P}_{\text {out }}$ curve grows closer to the MC curve as $d_{0} / r_{s}$ increases, because the quality of the lognormal approximation becomes more accurate as shown in Section 4.3. 


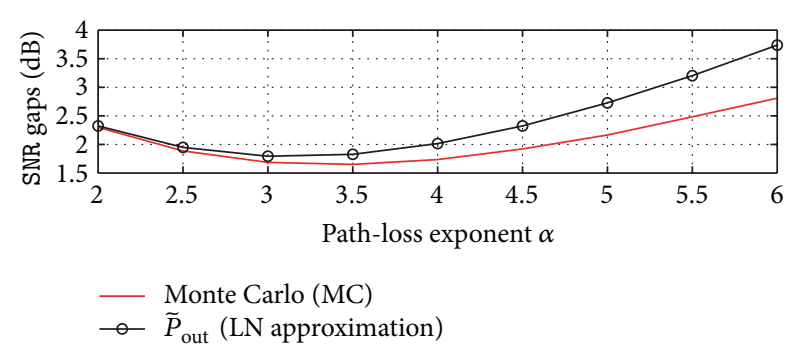

Figure 9: SNR gaps versus $\alpha$, when $r_{s}=1, d_{0}=2$, and $N=6$.

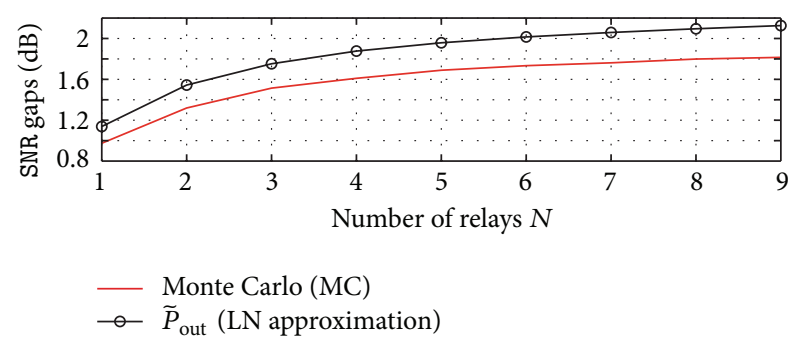

FIGURE 10: SNR gaps versus $N$, when $r_{s}=1, d_{0}=2$, and $\alpha=4$.

Figure 9 displays the impact of the path-loss exponent $\alpha$ on the SNR penalty, when $r_{s}=1, d_{0}=2$, and $N=6$. The two SNR gaps based on the MC and the LN approximation $\left(\widetilde{P}_{\text {out }}\right)$ have the convex curves that have the minimum heights at around $\alpha=3.5$. The height decreases in the two curves for $2 \leq$ $\alpha \leq 3.5$ are because the first-hop error relative to the secondhop error increases as $\alpha$ decreases for a fixed $d_{0} / r_{s}$, which means that the first-hop error cannot be ignored in this range. On the other hand, when $3.5 \leq \alpha \leq 6$, both curves increase, as $\alpha$ increases, because the path-loss disparity within the VMISO cluster becomes significant $\left(\sigma^{2}\right.$ and $\sigma_{l}^{2}$ are increasing functions of $\alpha$ ). Also, the height difference between the two curves increases as $\alpha$ increases. By testing first-hop error-free case $M=N$, we conclude that it is because the first-hop error, which is approximated by i.i.d. Bernoulli trials in Section 3.1, is overestimated. In other words, when $\alpha$ is large, the firsthop error relative to the second-hop error in the real outage (MC) is much smaller than the Bernoulli approximation, since $d_{0, k}^{\alpha} \ll d_{k, N+1}^{\alpha}$.

The last figure, Figure 10, shows the impact of the number of relays on the SNR gap, when $r_{s}=1, d_{0}=2$, and $\alpha=4$. As shown in the figure, the SNR gap increases as $N$ increases, because the path-loss disparity ignored in colocated approximation increases. Moreover, the height difference between the MC and the LN approximation is caused by the log-normal approximation error with the small $d_{0} / r_{s}=2$ as discussed in Section 4.3. As shown in Figure 8, for $d_{0} / r_{s}>2$, the gap between the two curves is smaller.

\section{Conclusion}

Motivated by the limited accuracy of the colocated assumption, which ignores the path-loss disparity in VMISO link, we analyze the impact of the path-loss disparity on the outage probability. Simulation results show that the SNR penalty (i.e., error) up to $3 \mathrm{~dB}$ should be considered, when the physically separated relays are assumed to be colocated. We investigate how the error changes depending on the system parameters such as $d_{0} / r_{s}, \alpha$, and $N$. Moreover, by the asymptotic analysis ( $\operatorname{SNR} \rightarrow \infty$ ) of the outage capacity, we show that the performance loss by the path-loss disparity has the same model as log-normal shadowing. The proposed outage approximation $\widetilde{P}_{\text {out }}$ is accurate enough to estimate the SNR gap (or the error of the colocated assumption) caused by the first-hop error and the path-loss disparity. Therefore, higher-layer protocol design and analysis with large-scale CT networks can be facilitated by the proposed approximation.

\section{Appendix}

Based on the asymptotic outage behavior in the high SNR regime, we propose the limit matching method that extracts the single parameter $\lambda$ by matching the limiting conditional outage rates of two distributions (13) and (14) as follows. First, when SNR $\rightarrow \infty$, the conditional CDF $F_{Y \mid M, \bar{\Lambda}_{d(1: M)}}(y)$ in (13) satisfies that

$$
\lim _{\operatorname{SNR} \rightarrow \infty} \frac{F_{Y \mid M, \bar{\Lambda}_{d(1: M)}}(g(\mathrm{SNR}, \mathrm{R}))}{(g(\operatorname{SNR}, \mathrm{R}))^{M+1}}=\frac{\prod_{k=0}^{M} \lambda_{k}}{(M+1) !} .
$$

On the other hand, the limiting $F_{Y \mid M, \lambda}(y)$ for the gamma distribution in (14) is given by

$$
\lim _{\operatorname{SNR} \rightarrow \infty} \frac{F_{Y \mid M, \lambda}(g(\text { SNR, R }))}{(g(\operatorname{SNR}, \mathrm{R}))^{M+1}}=\frac{\lambda^{M+1}}{(M+1) !} .
$$

Thus, if we match two limits (A.1) and (A.2), then we have

$$
\frac{\prod_{k=0}^{M} \lambda_{k}}{(M+1) !} \approx \frac{\lambda^{M+1}}{(M+1) !}
$$

which gives the projection equation in (16).

\section{Disclosure}

The material in this paper is presented in part at the International Conference on Communications (ICC), June 2013, Budapest, Hungary.

\section{Conflict of Interests}

The authors declare that there is no conflict of interests regarding the publication of this paper.

\section{Acknowledgment}

The authors gratefully acknowledge partial support for this research from the National Science Foundation, Grant no. CNS-1017984. 


\section{References}

[1] A. Sendonaris, E. Erkip, and B. Aazhang, "User cooperation diversity-part I: system description," IEEE Transactions on Communications, vol. 51, no. 11, pp. 1927-1938, 2003.

[2] J. N. Laneman, D. N. C. Tse, and G. Wornell, "Cooperative diversity in wireless networks: efficient protocols and outage behavior," IEEE Transactions on Information Theory, vol. 50, no. 12, pp. 3062-3080, 2004.

[3] A. Scaglione and Y.-W. Hong, "Opportunistic large arrays: cooperative transmission in wireless multihop ad hoc networks to reach far distances," IEEE Transactions on Signal Processing, vol. 51, no. 8, pp. 2082-2092, 2003.

[4] B. Sirkeci-Mergen, A. Scaglione, and G. Mergen, "Asymptotic analysis of multistage cooperative broadcast in wireless networks," IEEE Transactions on Information Theory, vol. 52, no. 6, pp. 2531-2550, 2006.

[5] L. V. Thanayankizil, A. Kailas, and M. A. Ingram, "Routing protocols for wireless sensor networks that have an opportunistic large array (OLA) physical layer," Ad-Hoc and Sensor Wireless Networks, vol. 8, no. 1-2, pp. 79-117, 2009.

[6] A. Kailas and M. A. Ingram, "Alternating opportunistic large arrays in broadcasting for network lifetime extension," IEEE Transactions on Wireless Communications, vol. 8, no. 6, pp. 28312835, 2009.

[7] H. Jung and M. A. Weitnauer, "Multi-packet interference in opportunistic large array broadcasts over disk networks," IEEE Transactions on Wireless Communications, vol. 12, no. 11, pp. 5631-5645, 2013.

[8] H. Jung and M. Weitnauer, "Multi-packet opportunistic large array transmission on strip-shaped cooperative routes or networks," IEEE Transactions on Wireless Communications, vol. 13, no. 1, pp. 144-158, 2014.

[9] T. R. Halford, K. M. Chugg, and A. Polydoros, "Barrage relay networks: System and protocol design," in Proceedings of the IEEE 21st International Symposium on Personal Indoor and Mobile Radio Communications (PIMRC '10), pp. 1133-1138, September 2010.

[10] Z. Zhou, S. Zhou, S. Cui, and J. Cui, "Energy-efficient cooperative communication in a clustered wireless sensor network," IEEE Transactions on Vehicular Technology, vol. 57, no. 6, pp. 3618-3628, 2008.

[11] G. Jakllari, S. V. Krishnamurthy, M. Faloutsos, and P. V. Krishnamurthy, "On broadcasting with cooperative diversity in multi-hop wireless networks," IEEE Journal on Selected Areas in Communications, vol. 25, no. 2, pp. 484-496, 2007.

[12] G. Jakllari, S. V. Krishnamurthy, M. Faloutsos, P. V. Krishnamurthy, and O. Ercetin, "A cross-layer framework for exploiting virtual MISO links in mobile ad hoc networks," IEEE Transactions on Mobile Computing, vol. 6, no. 6, pp. 579-594, 2007.

[13] S. Lakshmanan and R. Sivakumar, "Diversity routing for multihop wireless networks with cooperative transmissions," in Proceedings of the 6th Annual IEEE Communications Society Conference on Sensor, Mesh and Ad Hoc Communications and Networks (SECON '09), pp. 1-9, June 2009.

[14] J. W. Jung and M. A. Ingram, "Residual-energy-activated cooperative transmission (REACT) to avoid the energy hole," in Proceedings of the IEEE International Conference on Communications Workshops (ICC '10), May 2010.

[15] J. Lin and M. A. Ingram, "SCT-MAC: a scheduling duty cycle MAC protocol for cooperative wireless sensor network," in
Proceedings of the IEEE International Conference on Communications (ICC '12), pp. 345-349, IEEE, Ottawa, Canada, June 2012.

[16] A. Aksu and O. Ercetin, "Reliable multi-hop routing with cooperative transmissions in energy-constrained networks," IEEE Transactions on Wireless Communications, vol. 7, no. 8, pp. 2861-2865, 2008.

[17] A. Scaglione, D. L. Goeckel, and J. N. Laneman, "Cooperative communications in mobile ad hoc networks," IEEE Signal Processing Magazine, vol. 23, no. 5, pp. 18-29, 2006.

[18] B. Bash, D. Goeckel, and D. Towsley, "Clustering in cooperative networks," in Proceedings of the IEEE INFOCOM, pp. 486-490, April 2011.

[19] N. C. Beaulieu and J. Hu, "A closed-form expression for the outage probability of decode-and-forward relaying in dissimilar Rayleigh fading channels," IEEE Communications Letters, vol. 10, no. 12, pp. 813-815, 2006.

[20] J. N. Laneman, "Limiting analysis of outage probabilities for diversity schemes in fading channels", in Proceedings of the IEEE Global Telecommunications Conference (GLOBECOM '03), pp. 1242-1246, December 2003.

[21] S. Cho, W. Choi, and K. Huang, "QoS provisioning relay selection in random relay networks," IEEE Transactions on Vehicular Technology, vol. 60, no. 6, pp. 2680-2689, 2011.

[22] H. Wang, S. Ma, and T. Ng, "On performance of cooperative communication systems with spatial random relays," IEEE Transactions on Communications, vol. 59, no. 4, pp. 1190-1199, 2011.

[23] C. Zhai, W. Zhang, and G. Mao, "Uncoordinated cooperative communications with spatially random relays," IEEE Transactions on Wireless Communications, vol. 11, no. 9, pp. 3126-3135, 2012.

[24] V. Aalo, G. Efthymoglou, T. Soithong, M. Alwakeel, and S. Alwakeel, "Performance analysis of multi-hop amplify-andforward relaying systems in rayleigh fading channels with a poisson interference field," IEEE Transactions on Wireless Communications, vol. 13, no. 1, pp. 24-35, 2014.

[25] V. Aalo, K. Peppas, G. Efthymoglou, M. Alwakeel, and S. Alwakeel, "Serial amplify-and-forward relay transmission systems in nakagami-m fading channels with a poisson interference field," IEEE Transactions on Vehicular Technology, no. 99, p. 1, 2013.

[26] H. Wang, S. Ma, T. Ng, and H. V. Poor, "A general analytical approach for opportunistic cooperative systems with spatially random relays," IEEE Transactions on Wireless Communications, vol. 10, no. 12, pp. 4122-4129, 2011.

[27] T. K. Y. Lo, "Maximum ratio transmission," Proceedings of the IEEE International Conference on Communications (ICC '99), vol. 2, pp. 1310-1314, 1999.

[28] M.-K. Oh, X. Ma, G. Giannakis, and D.-J. Park, "Cooperative synchronization and channel estimation in wireless sensor networks," in Proceedings of the Conference Record of the 37th Asilomar Conference on Signals, Systems and Computers (ASILOMAR '03), vol. 1, pp. 238-242, November 2003.

[29] G. Barriac, R. Mudumbai, and U. Madhow, "Distributed beamforming for information transfer in sensor networks," in Proceedings of the 3rd International Symposium on Information Processing in Sensor Networks (IPSN '04), pp. 81-88, April 2004.

[30] R. Mudumbai, G. Barriac, and U. Madhow, "On the feasibility of distributed beamforming in wireless networks," IEEE Transactions on Wireless Communications, vol. 6, no. 5, pp. 1754-1763, 2007. 
[31] H. Jung and M. A. Weitnauer, "SNR penalty from the pathloss disparity in virtual multiple-input-single-output (VMISO) link," in Proceedings of the IEEE International Conference on Communications (ICC '13), pp. 5350-5354, June 2013.

[32] H. Jung, Y. J. Chang, and M. A. Ingram, "Experimental range extension of concurrent cooperative transmission in indoor environments at $2.4 \mathrm{GHz}$," in Proceedings of the 2010 IEEE Military Communications Conference (MILCOM '10), pp. 148153, November 2010.

[33] M. Abramowitz and I. Stegun, Handbook of Mathematical Functions with Formulas, Graphs, and Mathematical Tables, vol. 55, Dover, 1964.

[34] W. Choi and J. G. Andrews, "Downlink performance and capacity of distributed antenna systems in a multicell environment," IEEE Transactions on Wireless Communications, vol. 6, no. 1, pp. 69-73, 2007.

[35] S. M. Ross, Introduction to Probability Models, 2000.

[36] M. Abramowitz and I. A. Stegun, Handbook of Mathematical Functions 10th Printing with Corrections, Dover, New York, NY, USA, 1972.

[37] R. B. D’Agostino and M. A. Stephens, Goodness-of-Fit Techniques, vol. 68 of Statistics: Textbooks and Monographs, CRC Press, New York, NY, USA, 1986.

[38] E. Reusens, W. Joseph, B. Latré et al., "Characterization of onbody communication channel and energy efficient topology design for wireless body area networks," IEEE Transactions on Information Technology in Biomedicine, vol. 13, no. 6, pp. 933945, 2009.

[39] M. L. Sichitiu and V. Ramadurai, "Localization of wireless sensor networks with a mobile beacon," in Proceedings of the IEEE International Conference on Mobile Ad-Hoc and Sensor Systems, pp. 174-183, October 2004.

[40] M. Heidari, F. Akgul, and K. Pahlavan, "Identification of the absence of direct path in indoor localization systems," in Proceedings of the IEEE 18th International Symposium on Personal, Indoor and Mobile Radio Communications (PIMRC '07), pp. 1-6, Athens, Greece, September 2007.

[41] N. M. Razali and Y. B. Wah, "Power comparisons of shapirowilk, kolmogorov-smirnov, lilliefors and anderson-darling tests," Journal of Statistical Modeling and Analytics, vol. 2, no. 1, pp. 21-33, 2011. 

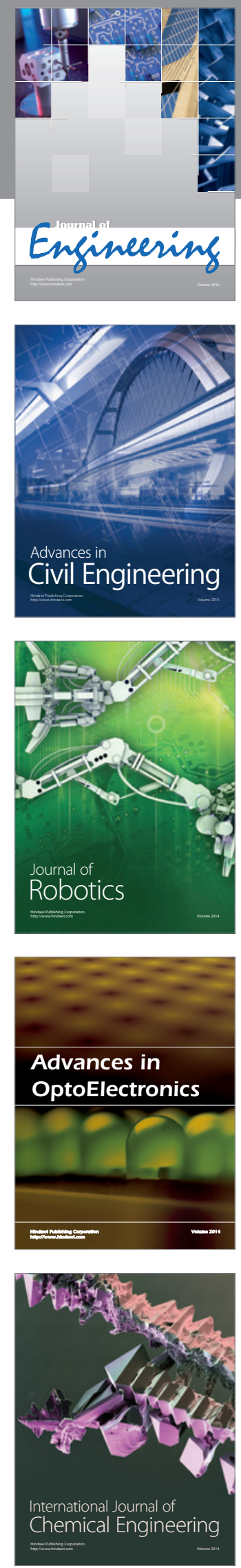

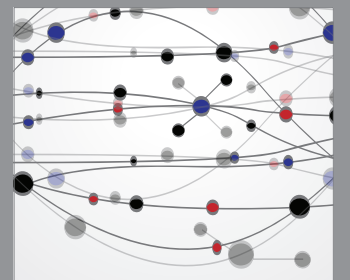

The Scientific World Journal
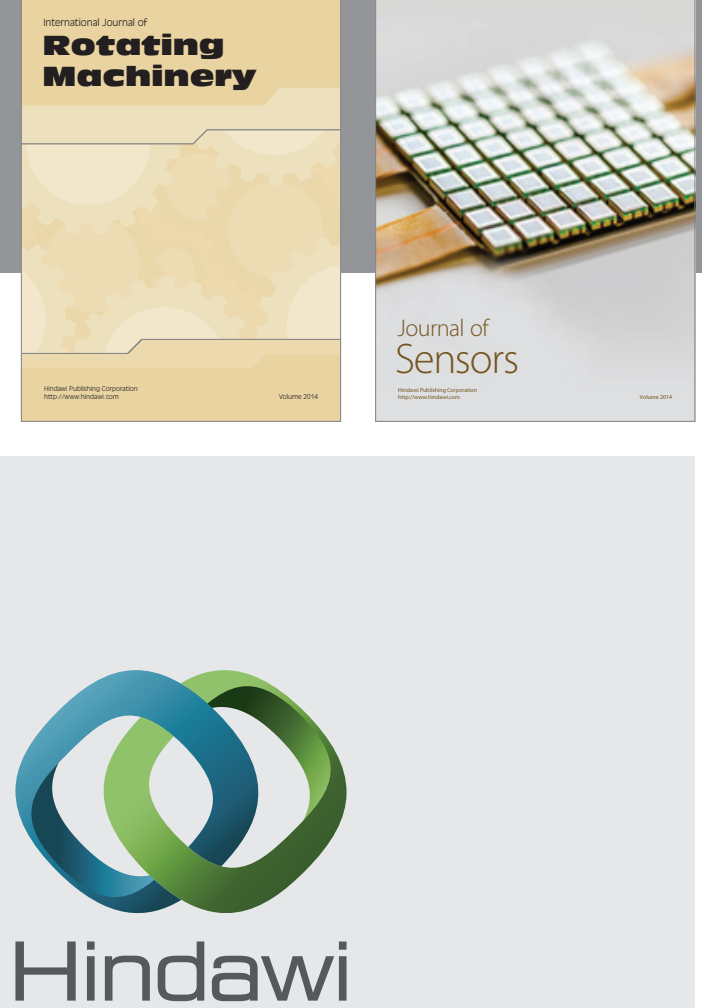

Submit your manuscripts at http://www.hindawi.com
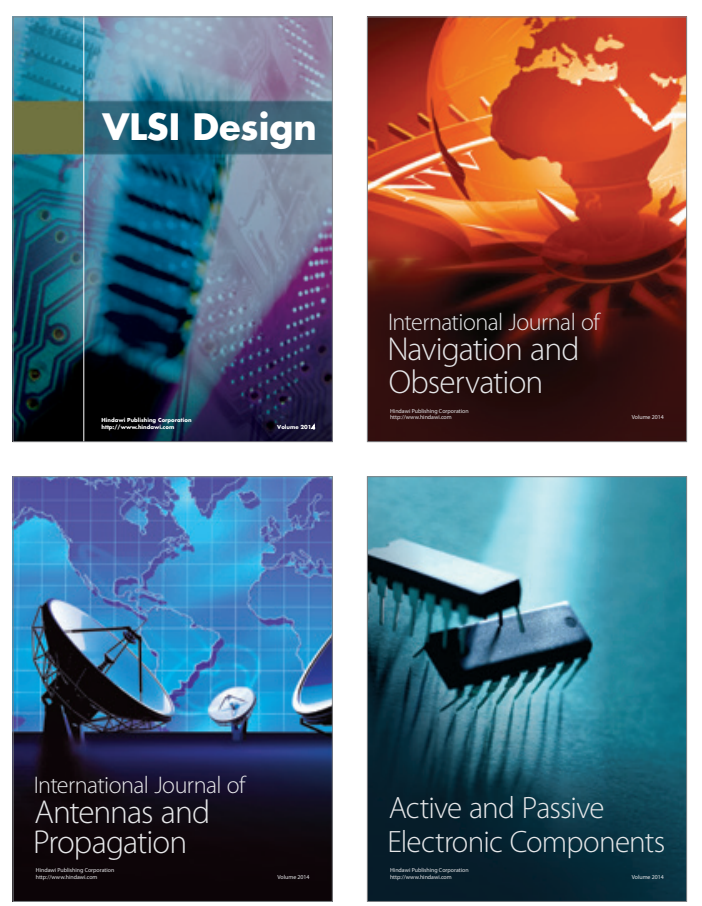
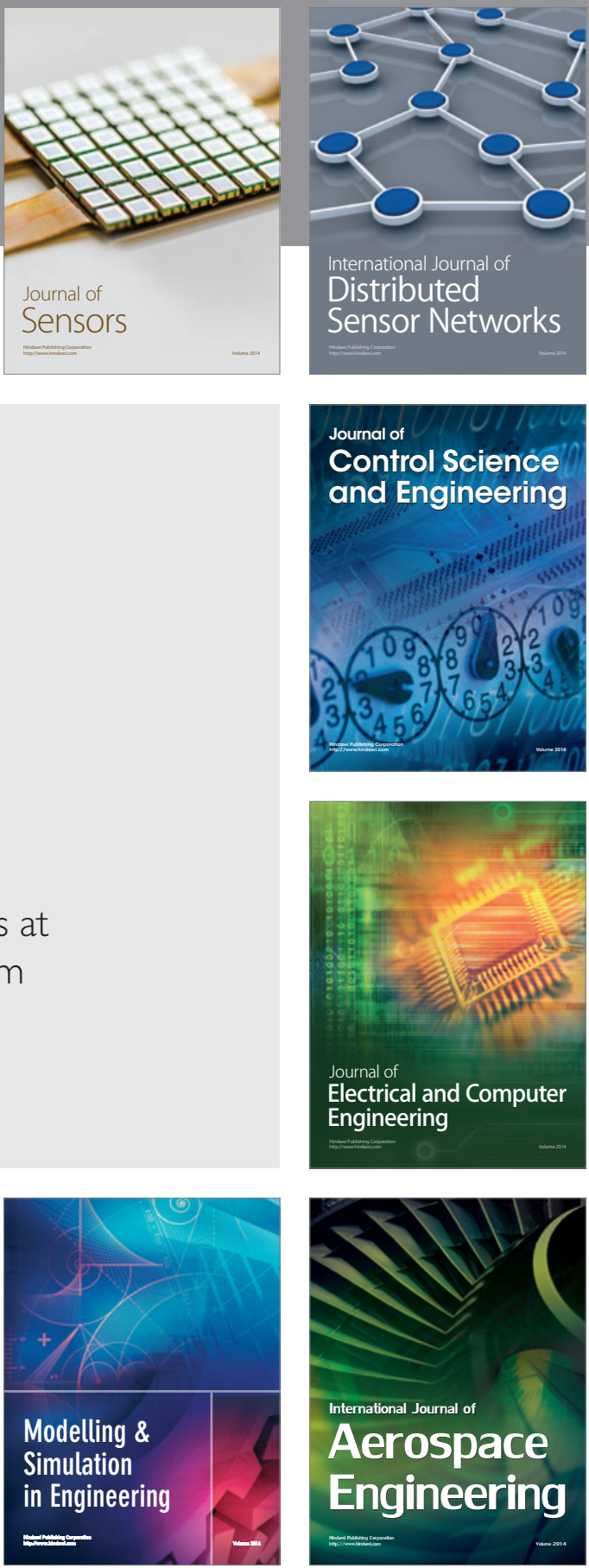

Journal of

Control Science

and Engineering
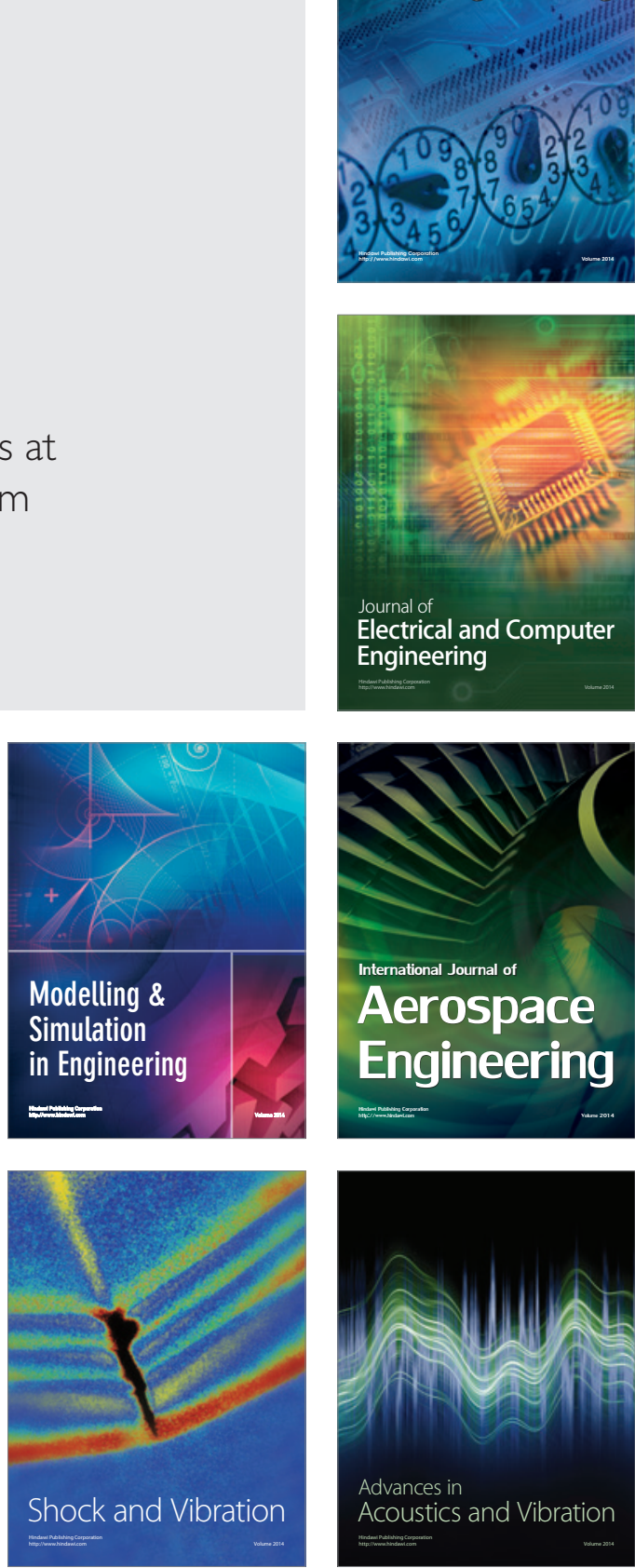